\title{
Identification of potential therapeutic targets for papillary thyroid carcinoma by bioinformatics analysis
}

\author{
MING ZHAO, KE-JING WANG, ZHUO TAN, CHUAN-MING ZHENG, ZHONG LIANG and JIAN-QIANG ZHAO \\ Department of Head and Neck Surgery, Zhejiang Cancer Hospital, Hangzhou, Zhejiang 310022, P.R. China
}

Received October 25, 2014; Accepted August 6, 2015

DOI: $10.3892 / 01.2015 .3829$

\begin{abstract}
The aim of the present study was to identify potential therapeutic targets for papillary thyroid carcinoma (PTC) and to investigate the possible mechanism underlying this disease. The gene expression profile, GSE53157, was downloaded from the Gene Expression Omnibus database. Only 10 chips, including 3 specimens of normal thyroid tissues and 7 specimens of well-differentiated thyroid carcinomas, were analyzed in the present study. Differentially-expressed genes (DEGs) between PTC patients and normal individuals were identified. Next, Gene Ontology and Kyoto Encyclopedia of Genes and Genomes pathway analyses of DEGs were performed. Modules in the protein-protein interaction (PPI) network were identified. Significant target genes were selected from the microRNA (miRNA) regulatory network. Furthermore, the integrated network was constructed with the miRNA regulatory and PPI network modules, and key target genes were screened. A total of 668 DEGs were identified. Modules M1, M2 and M3 were identified from the PPI network. From the modules, DEGs of cyclin-dependent kinase inhibitor 1A, S100 calcium binding protein A6 (S100A6), dual specificity phosphatase 5 , keratin 19 , met proto-oncogene (MET) and lectin galactoside-binding soluble 3 were included in the Malacards database. In the miRNA regulatory and integrated networks, genes of cyclin-dependent kinase inhibitor 1C (CDKN1C), peroxisome proliferator-activated receptor $\gamma$, aryl hydrocarbon receptor, basic helix-loop-helix family, member e40 and reticulon 1 were the key target genes. S100A6, MET and CDKN1C may exhibit key roles in the progression and development of PTC, and may be used as specific therapeutic targets in the treatment of PTC. However, further experiments are required to confirm these results.
\end{abstract}

Correspondence to: Dr Ke-Jing Wang, Department of Head and Neck Surgery, Zhejiang Cancer Hospital, 38 Guangji Road, Hangzhou, Zhejiang 310022, P.R. China E-mail: kejing_wdr@163.com

Key words: papillary thyroid carcinoma, differentially-expressed gene, modules

\section{Introduction}

As the most common thyroid malignancy worldwide, papillary thyroid carcinoma (PTC) accounts for $\sim 80 \%$ of all thyroid cancers (1). The incidence rate of PTC exhibits the most rapid increase of all cancers among women, and the second most rapid increase among men (2). Despite the advances in surgery, $\sim 5 \%$ of patients with PTC experience recurrence within 5 years of the initial treatment (3). Understanding the molecular mechanism involved in the proliferation, apoptosis and invasion of PTC is extremely important for the development of more effective therapeutic strategies.

Recently, certain differentially-expressed genes (DEGs) have been reported to exhibit important roles in PTC, and identification of these may be useful in the investigation of the molecular mechanisms of PTC $(4,5)$. Previous studies showed that ret proto-oncogene, neurotrophic tyrosine kinase receptor type 1 and v-raf murine sarcoma viral oncogene homolog B may be useful therapeutic targets for PTC (6-8). Ye et al found that Krüppel-like factor 17 may serve as a candidate tumor suppressor and a therapeutic target in PTC (9). Programmed cell death 4 was reported to exhibit an inhibitory role in the cell proliferation, malignant progression and invasion of PTC (10). microRNA (miRNA/miR)-199b-5p, miR-30a-3p and miR-146b-5p may be associated with PTC invasiveness (11). Although serious attempts have been made to find novel targets for gastric cancer treatment, at present, this knowledge is insufficient.

In the present study, DEGs between PTC patients and normal individuals were identified. Modules were then screened from the protein-protein interaction (PPI) network and the significant target genes were selected from the miRNA regulatory network. Through the identification of key genes, the possible molecular mechanism and potential therapeutic targets for PTC were investigated.

\section{Materials and methods}

Affymetrix microarray data. The gene expression profile, GSE53157, which was deposited by Pita et al (12), was obtained from the Gene Expression Omnibus database (http://www.ncbi.nlm.nih.gov/geo/). Gene expression profiling was based on the platform of GPL570 ([HG-U133_Plus_2] Affymetrix Human Genome U133 Plus 2.0 Array). The array consists of 54,675 probesets that can be used to detect the 
transcription level of 18,750 human genes. Only 10 chips, including 3 specimens of normal thyroid tissues and 7 specimens of well-differentiated thyroid carcinomas, were analyzed in the present study.

Identification of DEGs. The raw data were first preprocessed using the Affy package (13) in R language. Next, DEGs between normal thyroid tissues and well-differentiated thyroid carcinomas were analyzed by limma package in $\mathrm{R}$ (14). Fold-change (FC) of the expression of individual genes was also calculated for the differential expression test. DEGs with an adjusted P-value (Adj.P.Val) of $<0.05$ and $\mid \log \mathrm{FCl} \geq 1$ were considered to be significant. Adj.P.Val was the result of multiple testing corrections using the Benjamini-Hochberg (HB) method (15).

Gene Ontology (GO) and pathway enrichment analysis of $D E G s$. The GO analysis has become a commonly used approach for functional studies of large-scale transcriptomic or genomic data (16). The Kyoto Encyclopedia of Genes and Genomes (KEGG) pathway database (17) contains information of how molecules or genes are networked. The Database for Annotation Visualization and Integrated Discovery (DAVID) (18) was used to systematically extract biological meaning from large gene or protein lists. The GO function and KEGG pathway of DEGs were analyzed using DAVID 6.7 with FDR $<0.05$.

Construction of PPI network and screening of module. Genes associated with PTC were downloaded from the Malacards database (19). The downloaded genes and the identifided DEGs were then combined; the pooled dataset is referred to as PTC-associated genes in the present study. The Search Tool for the Retrieval of Interacting Genes (STRING) (20) database was used to retrieve the predicted interactions for the PTC-associated genes; version 9.1 of STRING covers 1,133 completely sequenced species. All associations obtained in STRING are provided with a confidence score, and each score represents a rough estimate of how likely a given association describes a functional linkage between two proteins (21). The PTC-associated genes with a confidence score of $>0.4$ were selected to construct the PPI network, using the Cytoscape software (22).

Visualizing complex networks and integrating these networks to any type of attribute data was allowed by Cytoscape (http://cytoscape.org/). The clusterMaker 1.11 (23) plugin in Cytoscape and the Markov cluster algorithm (24) were used to divide the PPI into modules (granularity parameter, 2). GO functional analysis of the genes in the modules was performed using the BinGo 2.44 plugin in Cytoscape (25) with a threshold of $\mathrm{P}<0.05$ using the hypergeometric test.

Enrichment analysis of microRNA targets. The PTC-associated microRNAs were screened from genes that were obtained from the Malacards database (19). The identified DEGs were then submitted into a web-based gene set analysis toolkit (WebGestalt) (26), and miRNA-target gene enrichment analysis was performed. The enrichment significance of the predicted target genes in the DEGs was tested using hypergeometric distribution. The target genes with an adj.P.Val of $<0.01$ were considered to be significant, which was the result of multiple testing correction using the HB method (15). Next, miRNA regulatory networks were built using Cytoscape (25). Furthermore, the integrated network was constructed using the miRNA regulatory network and the PPI modules.

\section{Results}

$G O$ and pathway enrichment analysis of DEGs. In total, 668 DEGs, including 262 upregulated genes and 406 downregulated genes, were selected. Results of GO analysis showed that the upregulated DEGs were significantly enriched in biological processes such as the regulation of protein kinase activity, the regulation of transferase activity and the induction of programmed cell death (Table I). Results of pathway analysis showed that the upregulated DEGs were significantly enriched in the p53 signaling pathway (Table I). However, there were no significant GO biological processes and pathways in the downregulated DEGs.

Module screening from the PPI network. A total of 355 genes asscoiated with PTC were downloaded from the Malacards database. These genes and the identified DEGs were combined, and 983 PTC-associated genes were obtained. The PPI network was constructed based on the predicted interactions of the 983 PTC-associated genes. A total of 1,157 unique PPI pairs and 413 nodes were included. The PPI network was divided into three modules (Fig. 1A-C). Tumor protein p53 (TP53) was the core of module 1, which included 55 unique PPI pairs and 31 nodes. In module 1, the upregulated genes of cyclin-dependent kinase inhibitor 1A and S100 calcium binding protein A6 (S100A6) were included in the Malacards database (Fig. 1A). V-raf-1 murine leukemia viral oncogene homolog 1 was the core of module 2 , and a total of 45 unique PPI pairs and 26 nodes were included in module 2 . In module 2 , the upregulated genes of dual specificity phosphatase 5 and keratin 19 were included in the Malacards database (Fig. 1B). Epidermal growth factor receptor was the core of module 3, and a total of 39 unique PPI pairs and 21 nodes were included in this module. In module 3 , the upregulated genes of met proto-oncogene (MET) and lectin galactoside-binding soluble 3 were included in the Malacards database (Fig. 1B). Modules 1, 2 and 3 were found to be significantly enriched $(\mathrm{P}<0.05)$ for one GO term each (Table II). The significant GO term in module 1 was the negative regulation of cellular metabolic process $\left(\mathrm{P}=8.16 \times 10^{-6}\right)$. The term response to chemical stimulus was the most significantly enriched function in module $2\left(\mathrm{P}=7.53 \times 10^{-7}\right)$. Notably, the transmembrane receptor protein tyrosine kinase signaling was the most significantly enriched pathway $\left(\mathrm{P}=6.50 \times 10^{-11}\right)$ in module 3 .

Enrichment analysis of microRNA target genes. The PTC-associated microRNAs acquired from the 355 genes were miR203, miR34b, miR221, miR222, miR146a, miR146b, miR21, miR181a1 and miR130b. Through miRNA-target gene enrichment analysis, a miRNA regulatory network consisting of 54 miRNAs and 210 DEGs was constructed (Fig. 2A). miR-221, miR-222, miR-181a, miR-203 and miR-130b, which were included in the 355 genes, were involved in the miRNA regulatory network. In addition, cyclin-dependent kinase inhibitor 1C (CDKN1C) was the target of miRNA-221 
Table I. GO and pathways analysis of the upregulated DEGs.

\begin{tabular}{llrl}
\hline Category & & \multicolumn{1}{c}{ Term } & Count \\
\hline GOTERM_BP_FAT & GO:0045859-regulation of protein kinase activity & 18 & $1.17 \times 10^{-2}$ \\
GOTERM_BP_FAT & GO:0045860-positive regulation of protein kinase activity & 14 & $1.22 \times 10^{-2}$ \\
GOTERM_BP_FAT & GO:0043549-regulation of kinase activity & 18 & $1.36 \times 10^{-2}$ \\
GOTERM_BP_FAT & GO:0033674-positive regulation of kinase activity & 14 & $1.47 \times 10^{-2}$ \\
GOTERM_BP_FAT & GO:0051347-positive regulation of transferase activity & 15 & $1.51 \times 10^{-2}$ \\
GOTERM_BP_FAT & GO:0012502-induction of programmed cell death & 16 & $1.96 \times 10^{-2}$ \\
GOTERM_BP_FAT & GO:0010942-positive regulation of cell death & 19 & $2.16 \times 10^{-2}$ \\
GOTERM_BP_FAT & GO:0006917-induction of apoptosis & 16 & $2.35 \times 10^{-2}$ \\
GOTERM_BP_FAT & GO:0051338-regulation of transferase activity & 19 & $2.54 \times 10^{-2}$ \\
GOTERM_BP_FAT & GO:0043068-positive regulation of programmed cell death & 9 & $2.61 \times 10^{-2}$ \\
GOTERM_BP_FAT & GO:0043627-response to estrogen stimulus & $2.93 \times 10^{-2}$ \\
GOTERM_BP_FAT & GO:0043065-positive regulation of apoptosis & $9.20 \times 10^{-2}$ \\
KEGG_PATHWAY & hsa04115:p53 signaling pathway & $9.46 \times 10^{-2}$
\end{tabular}

GO, Gene Ontology; DEG, differentially-expressed gene; BP, biological process; KEGG, Kyoto Encyclopedia of Genes and Genomes; count, number of DEGs in each GO term; FDR, false discovery rate.

Table II. GO terms of genes in three modules.

\begin{tabular}{|c|c|c|c|}
\hline Module & GO term & Corr. P & Genes in test set \\
\hline 1 & Negative regulation of cellular metabolic process & $8.16 \times 10^{-6}$ & $\begin{array}{l}\text { E2F1, CDKN1A, CDKN2A, PHB, } \\
\text { TRIM27, TP53, MDM2, TP63, } \\
\text { MDM4, BHLHE40,RUNX2, STAT3 }\end{array}$ \\
\hline 2 & Response to chemical stimulus & $7.53 \times 10^{-7}$ & $\begin{array}{l}\text { HNF 1A, BRAF, PMAIP1, BCL2L1, } \\
\text { SOD1, MAPK1, DUSP 4, KRT19, } \\
\text { KRAS, BAX, BCL2, MAPK3, } \\
\text { SCNN1G, SCNN1B, DUSP6 }\end{array}$ \\
\hline 3 & $\begin{array}{l}\text { Transmembrane receptor protein } \\
\text { tyrosine kinase signaling pathway }\end{array}$ & $6.50 \times 10^{-11}$ & $\begin{array}{l}P T P R J, E G F R, R E T, E R B B 3, \\
E R B B 2, G A B 1, P D G F R B, E G F, \\
N R G 1, G R B 7\end{array}$ \\
\hline
\end{tabular}

GO, Gene Ontology; Corr. P, corrected P-value.

and miRNA-222; v-kit Hardy-Zuckerman 4 feline sarcoma viral oncogene homolog, metallophosphoesterase domain containing 2 and peroxisome proliferator-activated receptor $\gamma$ were the targets of miRNA-130b; aryl hydrocarbon receptor was the target of miRNA-203; and dual specificity phosphatase 5 was the target of miRNA-203 and miRNA-181a (Fig. 2B). By combining the miRNA regulatory network with the PPI network modules, the integrated network was constructed (Fig. 3A-C). The upregulated gene basic helix-loop-helix family member e40 in module 1 was the target of miR-181a (Fig. 3A); and the downregulated gene reticulon 1 in module 2 was the target of miR-130b (Fig. 3B).

\section{Discussion}

PTC accounts for nearly $80 \%$ of human thyroid cancers, worldwide (27). Now, the burden of thyroid disease in the global population is enormous (28). Thus, the potential use of therapeutic targets appears to be the most promising area of research. In the present study, a bioinformatics approach was used to predict the potential therapeutic targets for PTC. The study identified 668 DEGs between normal thyroid tissues and well-differentiated thyroid carcinomas, among which, 406 genes were downregulated and 262 were upregulated. By constructing the PPI and miRNA regulatory networks, key genes were found, including S100A6, MET and CDKN1C.

S100A6 encodes a member of the S100A family of calcium binding proteins, and it is reported to be involved in the regulation of a wide range of cellular processes, including cell proliferation, differentiation and apoptosis $(29,30)$. Furthermore, S100A6 has been shown to be upregulated in numerous cancers, including thyroid carcinoma $(31,32)$. Li et al showed that increased S100A6 expression promoted the proliferation and migration of cells in human hepatocellular carcinoma (HCC), and that S100A6 may function as a potential therapeutic target in HCC (33). Komatsu et al showed that S100A6 was 


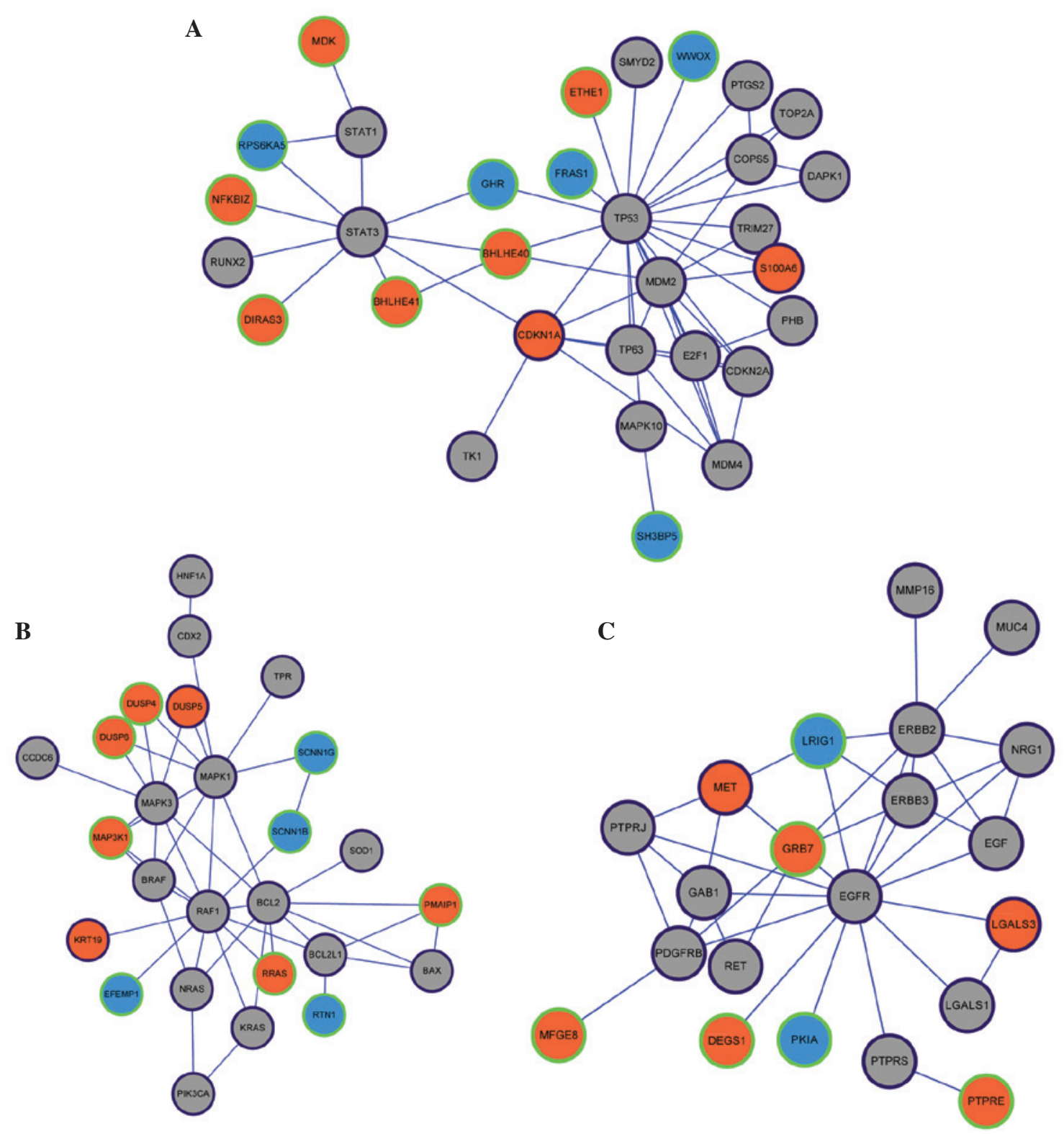

Figure 1. Selected modules from the protein-protein interaction network: (A) Module 1, (B) module 2 and (C) module 3. Orange and blue nodes represent products of up- and downregulated DEGs, respectively. Grey nodes represent PTC-associated genes that were not included in the DEGs. Nodes with a purple edge represent PTC-associated genes that were collected in the Malacard database. Nodes with a green edge represent PTC-associated genes that were not collected in the Malacard database. DEG, differentially-expressed gene; PTC, papillary thyroid carcinoma.

involved in the invasion and metastasis processes of human colorectal adenocarcinomas (34). S100A6 has been reported to be overexpressed in PTC and may contribute to certain events in this disease $(35,36)$. In the present study, S100A6 was overexpressed in PTC, and the PPI network showed that it had direct interaction with TP53, which was associated with PTC $(37,38)$. Overall, S100A6 may be a therapeutic target in PTC.

MET encodes the hepatocyte growth factor receptor and could regulate a number of physiological processes, including proliferation, scattering (cell dissociation and motility), morphogenesis and survival (39). MET could trigger tumor growth, angiogenesis and metastasis (40). Rong et al found that $M E T$ was overexpressed in a number of tumors, and suggested that MET activation contributed to tumor progression (41). Fujita and Sugano found that MET was overexpressed in primary colorectal cancer and liver metastases, and suggested that MET played an important role in the development of colorectal cancer liver metastases (42). In one study, Di Renzo et al showed that the overexpression of MET in human thyroid carcinomas may contribute to the progression of thyroid tumors (43), while in a second study, it was suggested that the upregulation of MET may add a selective growth advantage to differentiated ovarian cancers (44). In the present study, MET was upregulated in PTC, and GO analysis showed that it was involved in the biological process of transferase activity. Combined with the aforementioned studies, this data shows that MET may be a therapeutic target in PTC.

The putative tumor suppressor $C D K N 1 C$ is a negative regulator of cell proliferation and mutations. Soejima et al showed that $C D K N 1 C$ was downregulated in esophageal cancer and contributed to the tumor (45), and Hoffmann et al found that 

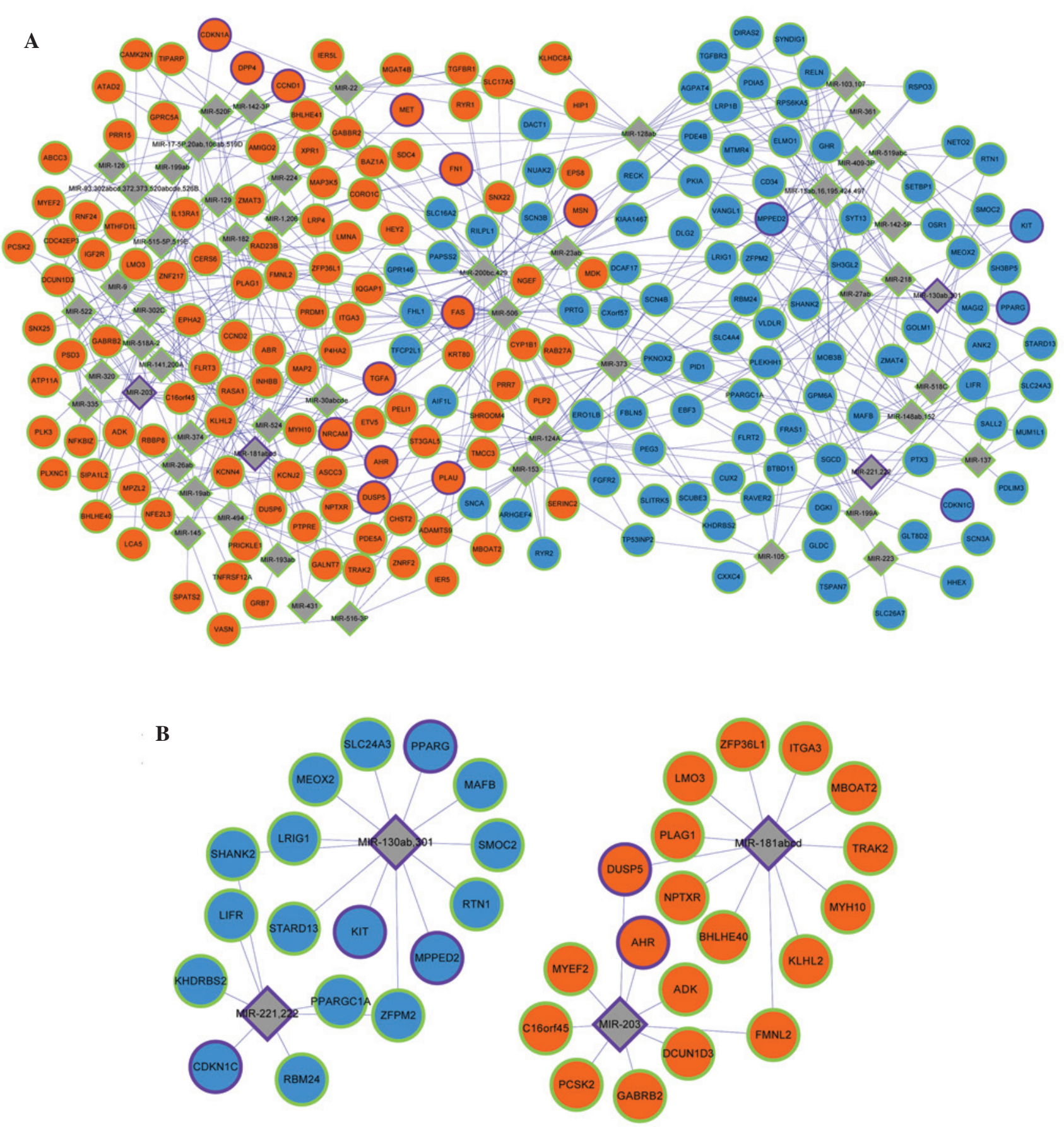

Figure 2. miRNA regulatory network. (A) miRNA regulatory network constructed with DEGs and their regulatory miRNAs. (B) miRNA regulatory network constructed with DEGs and their regulatory miRNAs. Diamond-shaped nodes represent miRNA and circular nodes represent DEGs. Orange and blue nodes represent products of up- and downregulated DEGs, respectively. Grey nodes represent PTC-associated genes that were not included in the DEGs. Nodes with a purple edge represent PTC-associated genes thatwere collected in the Malacard database; and nodes with a green edge represent PTC-associated genes that were not collected in the Malacard database. miRNA, microRNA; DEG, differentially-expressed gene; PTC, papillary thyroid carcinoma.

numerous advanced urothelial cancers displayed the downregulation of CDKNIC (46). Larson et al found that CDKNIC was a candidate tumor suppressor in breast cancer (47), while Algar et al found that it was a tumor suppressor in rhabdoid tumors (48). However, there have been no studies with regard to the association between $C D K N 1 C$ and PTC. In the present study, $C D K N 1 C$ was downregulated in PTC, and the miRNA regulatory network showed that $C D K N 1 C$ was the target of miRNA221 and miRNA222. One previous study showed that miRNA221 and miRNA222 were upregulated in PTC, and that they contributed to the procession of the disease (49). Furthermore, Fornari et al indicated that miR-221 controlled the expression of $C D K N 1 C$ in human hepatocellular carcinoma (50). Thus, $C D K N 1 C$ may be a potential therapeutic target in PTC.

In conclusion, S100A6, MET and CDKN1C may exhibit key roles in the progression and development of PTC, and may be used as specific therapeutic targets in the treatment of PTC. However, further experiments are required to confirm these results. 
A
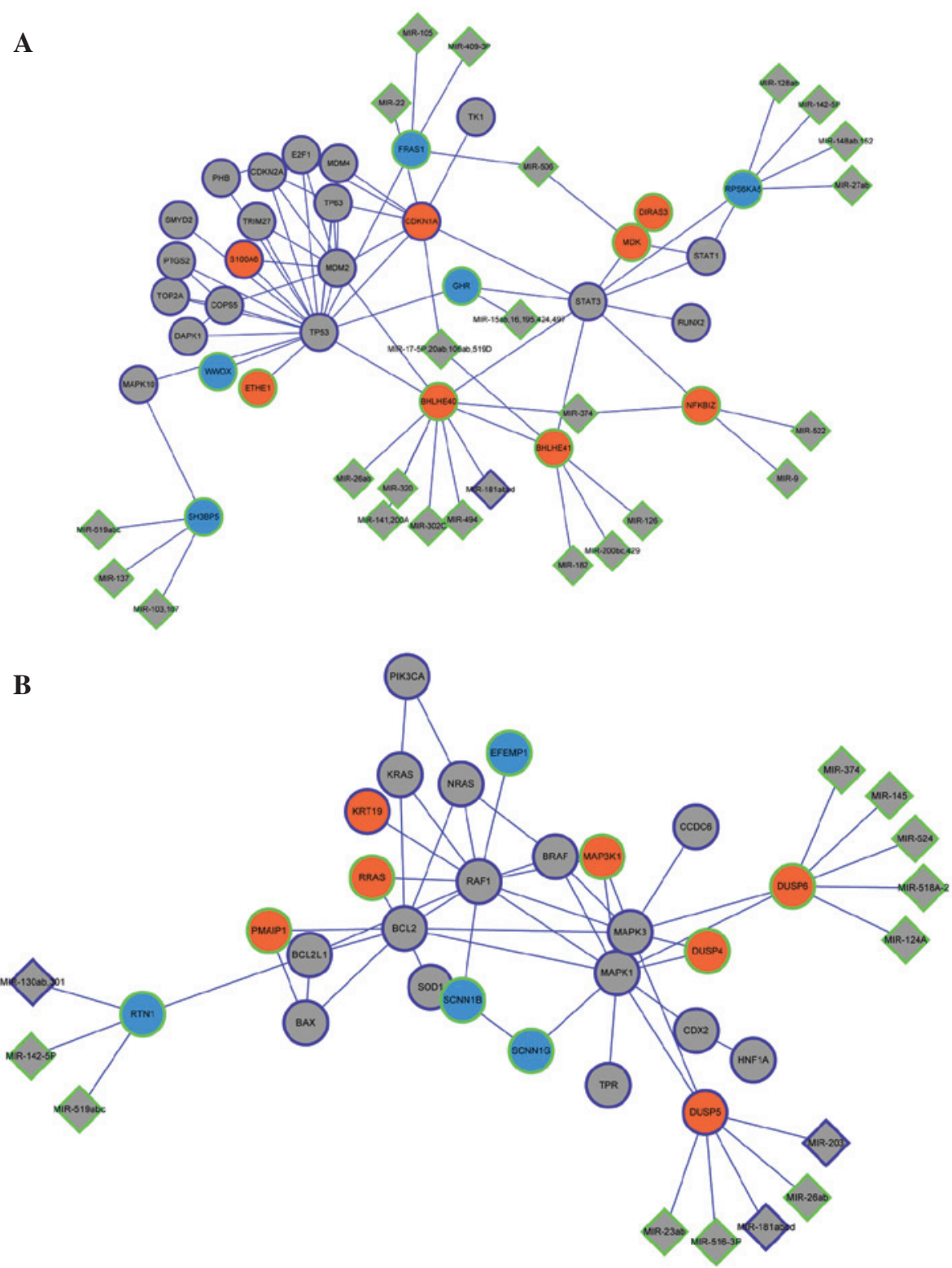

C

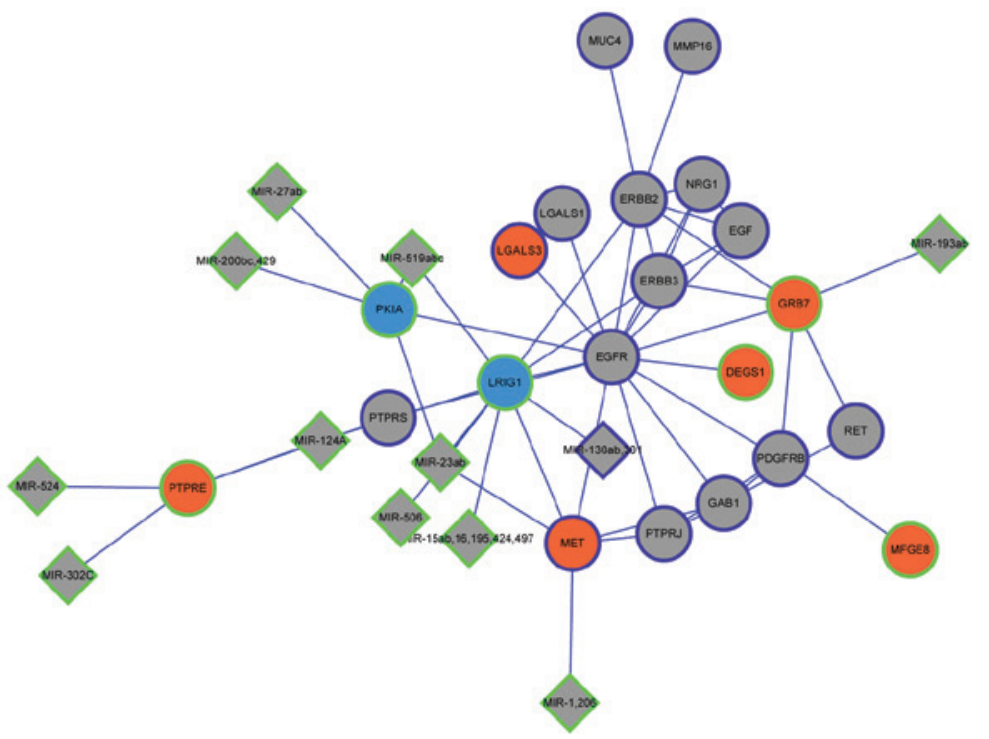

Figure 3. Integrated regulatory network. (A) Integrated network of module 1 and miRNA regulatory network. (B) Integrated network of module 2 and miRNA regulatory network. (C) Integrated network of module 3 and miRNA regulatory network. Diamond-shaped nodes represent miRNA and circular nodes represent DEGs. Orange and blue nodes represent products of up- and downregulated DEGs, respectively. Grey nodes represent PTC-associated genes that were not DEGs. Nodes with a purple edge represent PTC-associated genes that were collected in the Malacard database; and nodes with a green edge represent PTC-associated genes thatwere not collected in the Malacard database. miRNA, microRNA; DEG, differentially-expressed gene; PTC, papillary thyroid carcinoma. 


\section{Acknowledgements}

The authors wish to express gratitude to Fenghe (Shanghai) Information Technology Co., Ltd. (Shanhai, China) for ideas and assistance that provided a valuable added dimension to the present study.

\section{References}

1. Zhang J, Yang Y, Liu Y, Fan Y, Liu Z, Wang X, Yuan Q, Yin Y, Yu J, Zhu M, et al: MicroRNA-21 regulates biological behaviors in papillary thyroid carcinoma by targeting programmed cell death 4. J Surg Res 189: 68-74, 2014.

2. Siegel R, Naishadham D and Jemal A: Cancer statistics, 2012. CA Cancer J Clin 62: 10-29, 2012.

3. Grant CS: Recurrence of papillary thyroid cancer after optimized surgery. Gland Surg 4: 52-62, 2015.

4. Srivastava A, Goldberger H, Dimtchev A, Ramalinga M, Chijioke J, Marian C, Oermann EK, Uhm S, Kim JS, Chen LN, et al: MicroRNA profiling in prostate cancer-the diagnostic potential of urinary miR-205 and miR-214. PloS One 8: e76994, 2013.

5. Hodge LS, Elsawa SF, Grote DM, Price-Troska TL, Asmann YW, Fonseca R, Gertz MA, Witzig TE, Novak AJ and Ansell SM: MicroRNA expression in tumor cells from Waldenstrom's macroglobulinemia reflects both their normal and malignant cell counterparts. Blood Cancer J 1: e24, 2011.

6. Adeniran AJ, Zhu Z, Gandhi M, Steward DL, Fidler JP, Giordano TJ, Biddinger PW and Nikiforov YE: Correlation between genetic alterations and microscopic features, clinical manifestations and prognostic characteristics of thyroid papillary carcinomas. Am J Surg Pathol 30: 216-222, 2006.

7. Espinosa AV, Porchia L and Ringel MD: Targeting BRAF in thyroid cancer. Br J Cancer 96: 16-20, 2007.

8. Pelizzo MR, Boschin IM, Barollo S, Pennelli G, Toniato A, Zambonin L, Vianello F, Piotto A, Ide EC, Pagetta C, et al: BRAF analysis by fine needle aspiration biopsy of thyroid nodules improves preoperative identification of papillary thyroid carcinoma and represents a prognostic factor. A mono-institutional experience. Clin Chem Lab Med 49: 325-329, 2011.

9. Ye WC, Gao L, Huang J, Fang XM and Xie G: Suppressed Kruppellike factor 17 expression induces tumor proliferation, metastasis and a poor prognosis in papillary thyroid carcinoma. Mol Med Rep, 2014

10. Pennelli G, Fassan M, Mian C, Pizzi M, Balistreri M, Barollo S, Galuppini F, Guzzardo V, Pelizzo M and Rugge M: PDCD4 expression in thyroid neoplasia. Virchows Arch 462: 95-100, 2013.

11. Peng Y, Li C, Luo DC, Ding JW, Zhang W and Pan G: Expression profile and clinical significance of microRNAs in papillary thyroid carcinoma. Molecules 19: 11586-11599, 2014.

12. Pita JM, Banito A, Cavaco BM and Leite V: Gene expression profiling associated with the progression to poorly differentiated thyroid carcinomas. Br J Cancer 101: 1782-1791, 2009.

13. Gautier L, Cope L, Bolstad BM and Irizarry RA: affy-analysis of Affymetrix GeneChip data at the probe level. Bioinformatics 20: 307-315, 2004.

14. Smyth GK: Limma: Linear models for microarray data. In: Bioinformatics and Computational Biology Solutions Using $\{\mathrm{R}\}$ and Bioconductor. Gentleman R, Carey VJ, Huber W, Irizarry RA and Dudoit S (eds). Springer, New York, pp397-420, 2005.

15. Benjamini Y and Hochberg Y: Controlling the false discovery rate: A practical and powerful approach to multiple testing. J R Statist Soc B 57: 289-300, 1995.

16. Hulsegge I, Kommadath A and Smits MA: Globaltest and GOEAST: Two different approaches for gene ontology analysis. BMC Proc 3 (Suppl 4): S10, 2009.

17. Ogata H, Goto S, Sato K, Fujibuchi W, Bono H and Kanehisa M: KEGG: Kyoto encyclopedia of genes and genomes. Nucleic Acids Res 27: 29-34, 1999.

18. Dennis G Jr, Sherman BT, Hosack DA, Yang J, Gao W, Lane HC and Lempicki RA: DAVID: Database for annotation, visualization and integrated discovery. Genome Biol 4: P3, 2003.

19. Rappaport N, Nativ N, Stelzer G, Twik M, Guan-Golan Y, Stein TI, Bahir I, Belinky F, Morrey CP, Safran M and Lancet D: Mala Cards: An integrated compendium for diseases and their annotation. Database (Oxford) 2013: bat018, 2013.
20. Franceschini A, Szklarczyk D, Frankild S, Kuhn M, Simonovic M, Roth A, Lin J, Minguez P, Bork P, von Mering C and Jensen LJ: STRING v9.1: Protein-protein interaction networks, with increased coverage and integration. Nucleic Acids Res 41: D808-D815, 2013.

21. Szklarczyk D, Franceschini A, Kuhn M, Simonovic M, Roth A, Minguez P, Doerks T, Stark M, Muller J, Bork P, et al: The STRING database in 2011: Functional interaction networks of proteins, globally integrated and scored. Nucleic Acids Res 39: D561-D568, 2011.

22. Shannon P, Markiel A, Ozier O, Baliga NS, Wang JT, Ramage D, Amin N, Schwikowski B and Ideker T: Cytoscape: A software environment for integrated models of biomolecular interaction networks. Genome Res 13: 2498-2504, 2003.

23. Morris JH, Apeltsin L, Newman AM, Baumbach J, Wittkop T, Su G, Bader GD and Ferrin TE: ClusterMaker: A multi-algorithm clustering plugin for Cytoscape. BMC Bioinformatics 12: 436, 2011.

24. Enright AJ, Van Dongen S and Ouzounis CA: An efficient algorithm for large-scale detection of protein families. Nucleic Acids Res 30: 1575-1584, 2002.

25. Maere S, Heymans K and Kuiper M: BiNGO: A Cytoscape plugin to assess overrepresentation of gene ontology categories in biological networks. Bioinformatics 21: 3448-3449, 2005.

26. Wang J, Duncan D, Shi Z and Zhang B: WEB-based GEne SeT AnaLysis Toolkit (WebGestalt): Update 2013. Nucleic Acids Res 41: W77-W83, 2013.

27. Chen AY, Jemal A and Ward EM: Increasing incidence of differentiated thyroid cancer in the United States, 1988-2005. Cancer 115: 3801-3807, 2009.

28. Wang C and Crapo LM: The epidemiology of thyroid disease and implications for screening. Endocrinol Metab Clin North Am 26: 189-218, 1997.

29. Breen EC and Tang K: Calcyclin (S100A6) regulates pulmonary fibroblast proliferation, morphology and cytoskeletal organization in vitro. J Cell Biochem 88: 848-854, 2003.

30. Tsoporis JN, Izhar S and Parker TG: Expression of S100A6 in cardiac myocytes limits apoptosis induced by tumor necrosis factor-alpha. J Biol Chem 283: 30174-30183, 2008.

31. Cross SS, Hamdy FC, Deloulme JC and Rehman I: Expression of S100 proteins in normal human tissues and common cancers using tissue microarrays: S100A6, S100A8, S100A9 and S100A11 are all overexpressed in common cancers. Histopathology 46: 256-269, 2005

32. Ito Y, Yoshida H, Tomoda C, Uruno T, Miya A, Kobayashi K, Matsuzuka F, Kakudo K, Kuma K and Miyauchi A: Expression of S100A2 and S100A6 in thyroid carcinomas. Histopathology 46: $569-575,2005$

33. Li Z, Tang M, Ling B, Liu S, Zheng Y, Nie C, Yuan Z, Zhou L, Guo G, Tong A and Wei Y: Increased expression of S100A6 promotes cell proliferation and migration in human hepatocellular carcinoma. J Mol Med (Berl) 92: 291-303, 2014.

34. Komatsu K, Kobune-Fujiwara Y, Andoh A, Ishiguro S, Hunai H, Suzuki N, Kameyama M, Murata K, Miyoshi J, Akedo H, et al: Increased expression of S100A6 at the invading fronts of the primary lesion and liver metastasis in patients witholorectal adenocarcinoma. Br J Cancer 83: 769, 2000.

35. Ito Y, Yoshida H, Tomoda C, Uruno T, Miya A, Kobayashi K, Matsuzuka F, Kakudo K, Kuma K and Miyauchi A: Expression of S100A2 and S100A6 in thyroid carcinomas. Histopathology 46: $569-575,2005$.

36. Sofiadis A, Dinets A, Orre LM, Branca RM, Juhlin CC, Foukakis T, Wallin G, Höög A, Hulchiy M, Zedenius J, et al: Proteomic study of thyroid tumors reveals frequent up-regulation of the Ca2+-binding protein $5100 A 6$ in papillary thyroid carcinoma. Thyroid 20: 1067-1076, 2010.

37. Pita JM, Figueiredo IF, Moura MM, Leite V and Cavaco BM: Cell cycle deregulation and TP53 and RAS mutations are major events in poorly differentiated and undifferentiated thyroid carcinomas. J Clin Endocrinol Metab 99: E497-E507, 2014.

38. Quiros RM, Ding HG, Gattuso P, Prinz RA and Xu X: Evidence that one subset of anaplastic thyroid carcinomas are derived from papillary carcinomas due to BRAF and p53 mutations. Cancer 103: 2261-2268, 2005

39. Naldini L, Vigna E, Narsimhan RP, Gaudino G, Zarnegar R, Michalopoulos GK and Comoglio PM: Hepatocyte growth factor (HGF) stimulates the tyrosine kinase activity of the receptor encoded by the proto-oncogene c-MET. Oncogene 6: 501-504, 1991. 
40. Schmidt L, Junker K, Weirich G, Glenn G, Choyke P, Lubensky I, Zhuang Z, Jeffers M, Vande Woude G, Neumann H, et al: Two North American families with hereditary papillary renal carcinoma and identical novel mutations in the MET proto-oncogene. Cancer Rre 58: 1719-1722, 1998.

41. Rong S, Donehower LA, Hansen MF, Strong L, Tainsky M, Jeffers M, Resau JH, Hudson E, Tsarfaty I and Vande Woude GF: Met proto-oncogene product is overexpressed in tumors of p53-deficient mice and tumors of Li-Fraumeni patients. Cancer Res 55: 1963-1970, 1995.

42. Fujita $\mathrm{S}$ and Sugano K: Expression of c-met proto-oncogene in primary colorectal cancer and liver metastases. Jpn J Clin Oncol 27: 378-383, 1997.

43. Di Renzo M, Olivero M, Ferro S, Prat M, Bongarzone I, Pilotti S, Belfiore A, Costantino A, Vigneri R, Pierotti MA, et al: Overexpression of the c-MET/HGF receptor gene in human thyroid carcinomas. Oncogene 7: 2549-2553, 1992.

44. Di Renzo MF, Olivero M, Katsaros D, Crepaldi T, Gaglia P, Zola P, Sismondi P and Comoglio PM: Overexpression of the Met/HGF receptor in ovarian cancer. Int J Cancer 58: 658-662, 1994.

45. Soejima H, Nakagawachi T, Zhao W, Higashimoto K, Urano T, Matsukura S, Kitajima Y, Takeuchi M, Nakayama M, Oshimura M, et al: Silencing of imprinted CDKN1C gene expression is associated with loss of $\mathrm{CpG}$ and histone $\mathrm{H} 3$ lysine 9 methylation at DMR-LIT1 in esophageal cancer. Oncogene 23: 4380-4388, 2004.
46. Hoffmann MJ, Florl AR, Seifert $\mathrm{HH}$ and Schulz WA: Multiple mechanisms downregulate CDKN1C in human bladder cancer. Internat J Cancer 114: 406-413, 2005.

47. Larson PS, Schlechter BL, King CL, Yang Q, Glass CN, Mack C, Pistey R, de Las Morenas A and Rosenberg CL: CDKN1C/p57kip2 is a candidate tumor suppressor gene in human breast cancer. BMC Cancer 8: 68, 2008.

48. Algar EM, Muscat A, Dagar V, Rickert C, Chow CW, Biegel JA, Ekert PG, Saffery R, Craig J, Johnstone RW and Ashley DM: Imprinted CDKN1C is a tumor suppressor in rhabdoid tumor and activated by restoration of SMARCB1 and histone deacetylase inhibitors. PLoS One 4: e4482, 2009.

49. Tetzlaff MT, Liu A, Xu X, Master SR, Baldwin DA, Tobias JW, Livolsi VA and Baloch ZW: Differential expression of miRNAs in papillary thyroid carcinoma compared to multinodular goiter using formalin fixed paraffin embedded tissues. Endocr Pathol 18: 163-173, 2007.

50. Fornari F, Gramantieri L, Ferracin M, Veronese A, Sabbioni S, Calin GA, Grazi GL, Giovannini C, Croce CM, Bolondi L and Negrini M: MiR-221 controls CDKN1C/p57 and CDKN1B/p27 expression in human hepatocellular carcinoma. Oncogene 27: 5651-5661, 2008. 